\title{
Estimation of Length and Order of Polynomial-based Filter Implemented in the Form of Farrow Structure
}

\author{
Selena Vukotic \\ School of Computing \\ University Union \\ Belgrade 11000, Serbia \\ svukotic@raf.edu.rs
}

\author{
Dorde Babic \\ School of Computing \\ University Union \\ Belgrade 11000, Serbia \\ djbabic@raf.edu.rs
}

\begin{abstract}
Digital polynomial-based interpolation filters implemented using the Farrow structure are used in Digital Signal Processing (DSP) to calculate the signal between its discrete samples. The two basic design parameters for these filters are number of polynomial-segments defining the finite length of impulse response, and order of polynomials in each polynomial segment. The complexity of the implementation structure and the frequency domain performance depend on these two parameters. This contribution presents estimation formulae for length and polynomial order of polynomial-based filters for various types of requirements including attenuation in stopband, width of transitions band, deviation in passband, weighting in passband/stopband.
\end{abstract}

Keywords-digital filters; filter design; polynomial-based filters; Farrow structure

\section{INTRODUCTION}

The digital polynomial-based interpolation filters are used in signal processing applications whenever it is required to calculate signal samples at arbitrary positions between existing samples. The impulse response of digital polynomial-based interpolation filters is derived by using piecewise polynomial analogue model [1]. An underlying continuous-time impulse response $h_{a}(t)$ has the following properties [1]: first, $h_{a}(t)$ is nonzero only in a finite interval $0 \leq t \leq N T$ with $N$ being an integer which determines the length of the filter. Second, in each subinterval $n T \leq t<(n+1) T$, for $n=0,1 \ldots, N-1, h_{a}(t)$ is expressible as a polynomial of $t$ of a given order $M$. Third, $h_{a}(t)$ is symmetric with respect to its middle point $\mathrm{t}=N T / 2$, to guarantee phase linearity of the resulting overall system. The advantage of the above impulse response is in the fact that the actual implementation can be efficiently performed by using the Farrow structure [2] or its modifications such as transposed Farrow structure, prolonged Farrow structure etc [1, 3]. The number of multipliers in any modification of Farrow structure is directly proportional to polynomial order $M$ and number of

This work is supported by the Ministry of Education, Science and Technological Development of Republic of Serbia under technology development project: TR32028 - "Advanced Techniques for Efficient Use of Spectrum in Wireless Systems". polynomial segments $N$.

The polynomial-based interpolation filters can be designed using time domain or frequency domain design methods [1]. The time-domain design methods are based on Lagrange and B-spline interpolations, where the approximating polynomial is fitted to the discrete-time samples. In the frequency domain design methods, the coefficients of the polynomials, i.e. coefficient of the Farrow structure, are optimized directly in the frequency domain $[1,3]$. In all these design methods, there are two basic parameters that control performance of the filter and its complexity. These two basic parameters are the number of polynomial segments $N$ and polynomial order $M$.

As stated above, polynomial order $M$ and filter length $N$ are directly proportional to the number of multipliers in Farrow based structure. Thus, the system complexity measured in number of operations is directly related to these two parameters. Furthermore, the system performance in the frequency domain measured by stopband attenuation and passband ripple is also related to $N$ and $M$. Therefore, it is very important to estimate polynomial order $M$ and filter length $N$ according to given system parameters. This paper presents the estimation formulae for the length $N$ and polynomial order $M$ for various types of requirements. The formulae presented below can save time for the filter designers, because they get suitable starting values for $N$ and $M$ for the given set of requirements. They can serve to estimate the filter complexity for given set of system requirements.

Similar order estimation formulae exist for FIR filters, for example Kaiser order estimation [4]. In the actual digital implementation, polynomial-based filters can be modeled as FIR filters [1], thus we can use similar methodology for analysis. The estimation formula for $N$, which can be found in [5] is good starting point for filter length $N$. In [6], the estimation formulae for both $N$ and $M$ have been proposed. The work presented in [6], has been the main motivation for the research presented here. However, the formulae presented in [6] are obtained using trial and error method, and they have some conditional restrictions. Namely, the estimation formulae 
of [6] cannot be used in the case when filter with narrow transition band is designed. In [7], the estimation formulae for the prolonged Farrow structure has been proposed. Hence, in this paper we propose the more general and accurate formulae for estimation of polynomial order $M$ and filter length $N$ based on the system requirements including attenuation in stopband, width of transitions band, deviation in passband, weighting in passband/stopband.

\section{ESTIMATION OF POLYNOMIAL ORDER $M$ AND FILTER LENGTH $N$}

In the previous section, we have seen that the number of polynomial segments $N$ and the order $M$ of the polynomial are the design parameters that highly influence the performance of the filter in the frequency domain. Furthermore, the cost of realization, i.e. the number of multipliers, of a filter can be estimated by determining required values for $N$ and $M$. In this section, we present the estimation formulae and explain in detail an experimental method used to derive the formulae.

The estimation formula from [6] for the number of polynomial segments $N$ is rather similar to the Kaiser formula for order estimation of FIR filters [4], thus we can use it as good starting point. In this way, we can start from the Kaiser formula and adapt it to polynomial-based filters. Similarly to other approaches $[6,8]$, we use experimentally obtained results in order to determine the estimation formulae. To this end, a lot of filters were designed, by using different system specifications, in order to adapt the Kaiser formula to the polynomial-based case. The polynomial based filters are designed using the minimax optimization routine explained in [1] for the following design parameters. The passband edge is varied from $\Delta f_{p}$ to $F / 2-\Delta f_{p}$ with step equal to $\Delta f_{p}=0.05$ normalized to $F$, where $F$ is output/input sampling rate in decimation/interpolation case. The stopband edge $f_{s}$ is determined according to case A specification defined in [1], thus $f_{s}=0.5$ normalized to $F$. The weighting function $W(f)$, which is used to differentiate precision of design in passband and stopband, is also varied from $W(f)=\left[\begin{array}{ll}W_{p} & W_{s}\end{array}\right]=\left[\begin{array}{ll}1 & 0.1\end{array}\right]$ to $W(f)=\left[\begin{array}{ll}1 & 1000\end{array}\right]$, where $W_{p}$ and $W_{s}$ are weights in passband and stopband region respectively. Finally, we used different values for the number of polynomial segments $N$ ranging from $N=2$ to $N=24$ with step two, taking only even values of $N$. The polynomial order $M$ takes values from $M=0$ to $M=7$ with unity step. In this way, in our experiment we use 9 different values of $f_{p}$, five values of $W(f), 12$ values of $N$, and 8 values of $M$. All together, we have designed $9 \cdot 5 \cdot 12 \cdot 8=4320$ filters. For each set of requirements, we have determined obtained performance in terms of passband ripple $\delta_{p}$, and stopband ripple $\delta_{s}$.

Figures 1 and 2 illustrate the stopband attenuation as a function of used system parameters for Case A specifications from [8]. From Figure 1, one can see that for a given $M, f_{p}$, and $W(f)$ there is a certain value of $N$ after which the stopband attenuation $\delta_{s}$ saturates in value. Therefore, there is no point to design filters with higher $N$ for given set of $M, f_{p}$, and $W(f)$. From Figure 2, it is also possible to see that for given $N, f_{p}$, and $W(f)$ there is a certain value of $M$ after which the stopband attenuation $\delta_{s}$ saturates in value. Therefore, there is no point to design filters with higher $M$ for given set of $N, f_{p}$, and $W(f)$, as well. These border values of $N$ and $M$ are actually the optimal values to be used in design for given $\delta_{s}, f_{p}$, and $W(f)$. Therefore, we take these border values for $N$ with appropriate system parameters to curve fitting toolbox assuming the same type of function as in the case of Kaiser estimation formula. The starting formula can be represented as:

$$
-20 \log _{10}\left(\delta_{s}\right)=a \cdot N+b
$$

with coefficients $a$ and $b$, which should be determined.
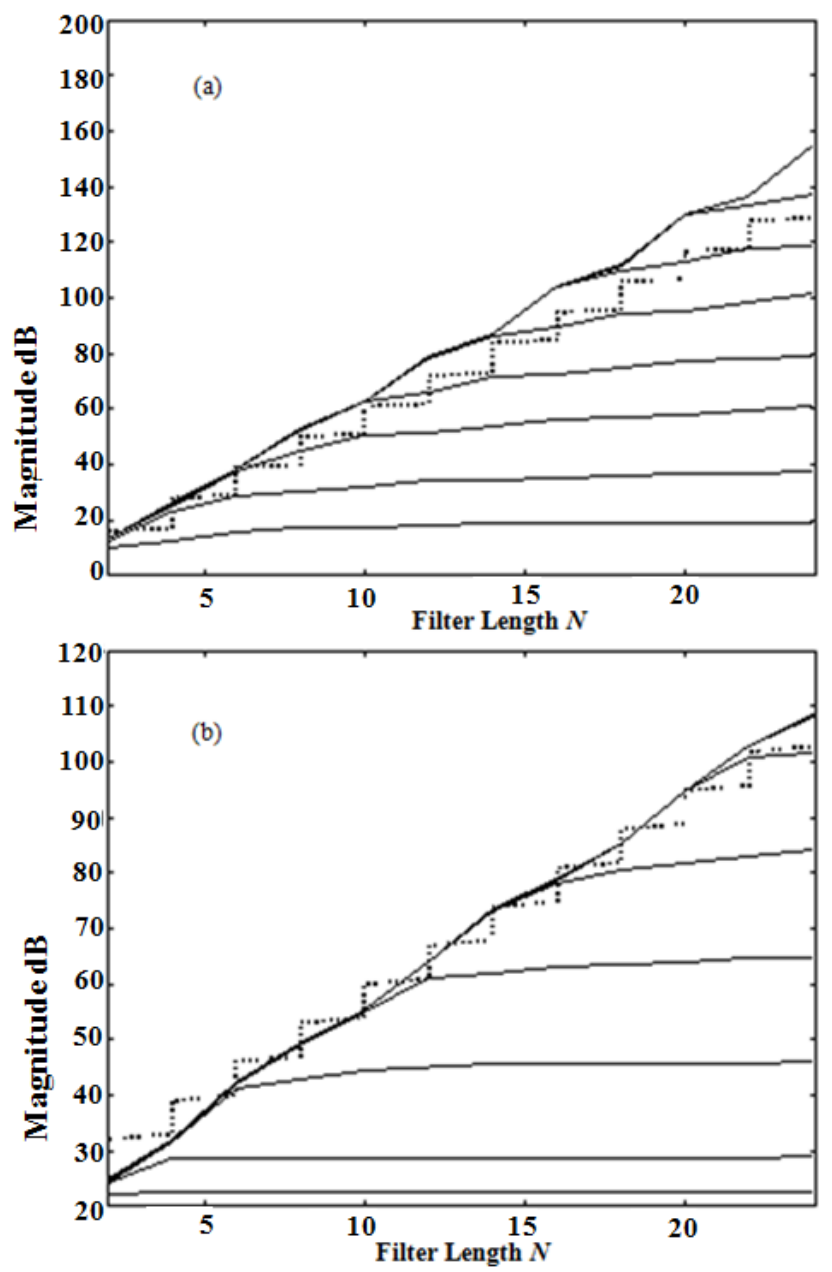

Fig. 1. Case A specifications: The curves are shown for $M$ equals 0 to 7 . Dashed line is obtained from the estimation formula for $N$ shown in (2). The stopband edge is at $f_{s}=0.5$ normalized to $F$, passband edge and stopband weighting are at: (a) $f_{p}=0.15 F$ and $W=0.1$; (b) $f_{p}=0.3 F$ and $W=10$.

The curve fitting toolbox is applied with experimental data, and the following estimation formula is obtained:

$$
N=2 \cdot\left[0.5 \cdot\left(\left(\frac{-20 \log _{10}\left(\delta_{s}\right)}{28 \cdot\left(f_{s}-f_{p}\right)}\right)+\left(\frac{0.45 \cdot w}{\left(f_{s}-f_{p}\right)}\right)-0.5 \cdot w\right)\right]
$$

where $\delta_{p}$ and $\delta_{s}$ are the maximum deviations of the amplitude response from unity for $f \in\left[0, f_{p}\right]$ and the maximum deviation from zero in stopbands, respectively, and $w=1-\log _{10}(W)$. Here, 
$\lceil x\rceil$ stands for the smallest integer which is larger or equal to $x$. It has been observed that in the most cases the above estimation formula is rather accurate. However, if the transition band is narrow, i.e., in the case when $\left(f_{s}-f_{p}\right) / F \leq 0.1$, the required value of $N$ should be increased by 2 . A dashed line in Figure 1 is a plot obtained from the estimation formula for $N$ shown in (2). One can see that the dashed line represents the border values of $N$ when performance saturates as explained above.
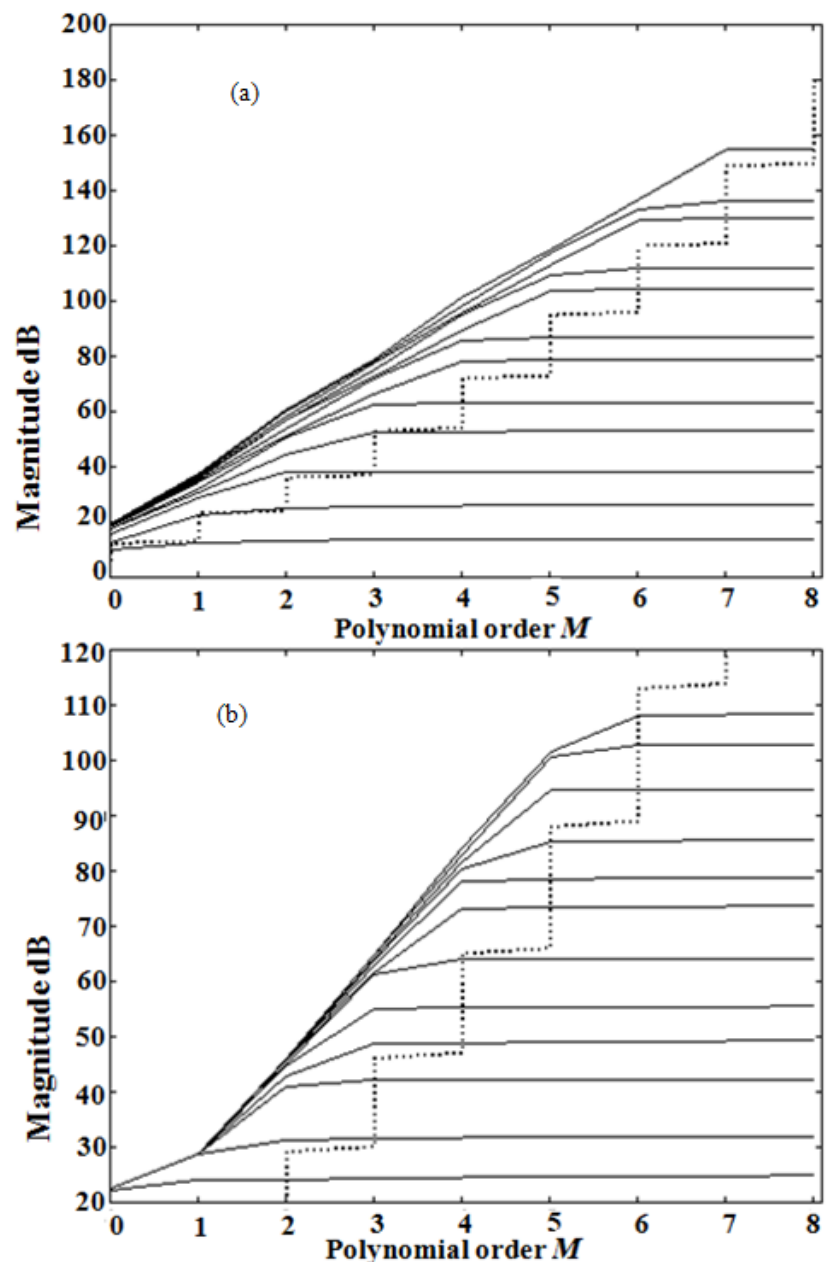

Fig. 2. Case A specifications: The curves are shown for $N$ equals 2 to 24 Dashed line is obtained from the estimation formula for $M$ shown in (4). The stopband edge is at $f_{s}=0.5$, passband edge and stopband weighting are at (a) $f_{p}=0.15 F$ and $W=0.1$; (b) $f_{p}=0.3$ Fand $W=10$.

The next problem is to find the minimum value of the polynomial order $M$ to meet the specifications, which is actually border value of $M$ in Figure 2 when performance saturates. It has been observed that the required value of $M$ depends on the type of requirements. Never the less, it is possible to consider the following estimate as a good starting point for all three types of requirements:

$$
-20 \cdot \log _{10}\left(\delta_{s}\right)=c \cdot M^{2}+d \cdot M+e
$$

The coefficients $c, d$ and $e$ are adjusted using experimentally obtained data within curve fitting toolbox. The Case A estimation formula can be expressed as:

$$
M=\left\lceil\sqrt{\frac{A_{s}+\left(10 \log _{10}(W)-40\right) \cdot \Delta f+10}{1.5}+\frac{0.13}{\Delta f}}\right\rceil-3
$$

where $\Delta f=\left(f_{s}-f_{p}\right)$. It has been observed that if transition band is relatively large to the sampling frequency, that is when $\left(f_{s}-f_{p}\right) / F$ $\geq 0.5$, the required value of polynomial order $M$ can be lowered by one. The estimation formula cannot be used when the transition band is very small, i.e., in the case when $\left(f_{s}-f_{p}\right) / F$ $<0.1$. (Lower bound for the transition bandwidth in the experiment is $\left(f_{s}-f_{p}\right) / F=0.1$, thus we do not have data for the case when $\left.\left(f_{s}-f_{p}\right) / F<0.1\right)$. A dashed line in Figure 2 is a plot obtained from the estimation formula for $M$ shown in (4). One can see that the dashed line represents the border values of $N$ when performance saturates as explained above.

\section{Design EXAMPLES}

This section gives an example in order to illustrate how to use the presented formulae. At the same time we can estimate the performance of the proposed estimation formulae. The following system requirements are considered: passband and stopband edges are at $f_{p}=0.4 F$ and at $f_{s}=0.5 F$, the filter is to be designed in minimax sense with stopband attenuation $A_{s}=60 \mathrm{~dB}$, passband weighting equal to unity and stopband weightings of $W=100$. In order to design the filter according to the specifications given above, first, we use (2) to estimate the number of intervals for the Case A specifications, and we obtain $N=18$. The degree of the polynomial in each subinterval $M=4$ is estimated using (4). We design filter using obtained $N$ and $M$ with earlier defined system requirements. Figure 3 gives the frequency response for Case A filter with achieved stopband attenuation $A_{s}=59.5 \mathrm{~dB}$ and passband ripple $\delta_{p}=0.1065$ in linear scale. It can be observed that the estimation formulae are relatively good, as they estimate the border performance for the given set of requirements.

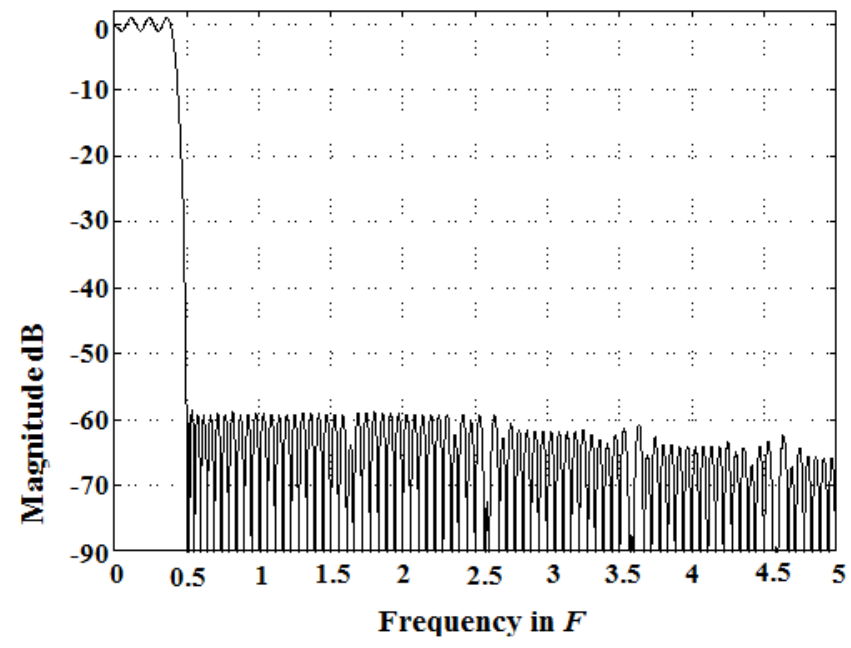

Fig. 3. Design Example: The frequency domain performance of filter whose parameters are estimated using the presented formulae. The filter specifications are: stopband attenuation $A_{s}=60 \mathrm{~dB}$, passband weighting equal to unity and stopband weighting is $W=100$, passband edge $f_{p}=0.4 F$. Case A filter of length $N=18$, and $M=4$ with achieved $A_{s}=59.5 \mathrm{~dB}$ and $\delta_{p}=0.1065$. 


\section{CONCLUSION}

In this paper, the estimation formulae for the number of polynomial segments $N$ and the polynomial order $M$ of polynomial-based filter are presented. It has been shown that these estimates give the border performance of the filter for the given set of specifications. Formulae for $N$ and $M$ can be used to estimate the starting value of these two parameters in minimax optimization used to design the Farrow structure. This is significant information which can save time for filter designers. Furthermore, the formulae for $N$ and $M$ can be used to estimate implementation costs of the Farrow based filters for the given set of requirements. The formulae can also be used to estimate implementation costs of multistage sampling rate converters containing Farrow filter. Estimation formulae for Case $\mathrm{B}$ and Case $\mathrm{C}$ requirements with minimax optimization, as well as, least-mean-square optimization are part of future work.

\section{References}

[1] J. Vesma, T. Saramaki, "Polynomial-based interpolation Filters-Part I: Filter synthesis", Circuits, Systems, and Signal Processing, Vol. 26, No 2, pp. 115-146, 2007

[2] C. W. Farrow, "A continuously variable digital delay element", IEEE International Symposium on Circuits and Systems, pp. 2641-2645, Espoo, Finland, June 7-9, 1988

[3] D. Babic, T. Saramaki, M. Renfors, "Conversion between arbitrary sampling rates using polynomial-based interpolation filters", 2nd International TICSP Workshop on Spectral Methods and Multirate Signal Processing, pp. 57-64, Toulouse, France, 2002

[4] J. F. Kaiser, "Nonrecursive digital filter design using the I0-sinh window function", IEEE International Symposium on Circuits and Systems, pp. 20-23, 1974

[5] T. Saramaki, Multirate signal processing, Lecture Notes, Tampere University of Technology, Finalnd, 2012 available at: http://www.cs.tut.fi/ ts/

[6] D. Babic, H. G. Gockler, "Estimation of the length and the polynomial order of polynomial-based filters", 8th International Conference on Sampling Theory and Applications, Marseille, France, May 18-22, 2009

[7] D. Babic, "Estimation of the number of polynomial segments and the polynomial order of prolonged Farrow structure", 22nd Telecommunications Forum Telfor (TELFOR), Belgrade, Serbia, pp. 461 - 464, November 25-27, 2014

[8] T. Saramaki, "Finite impulse response filter design", in Handbook for Digital Signal Processing, John Wiley \& Sons, New York, USA, 1993 